\title{
Radiotherapy for gastric mucosa-associated lymphoid tissue lymphoma: dosimetric comparison and risk assessment of solid secondary cancer
}

\author{
Sun Hyun Bae, MD, PhD ${ }^{1,2}$, Dong Wook Kim, PhD 3 , Mi-Sook Kim, MD, PhD4, Myung-Hee Shin, PhD ${ }^{5}$, \\ Hee Chul Park, MD, PhD², Do Hoon Lim, MD, PhD² \\ 'Department of Radiation Oncology, Soonchunhyang University Bucheon Hospital, Bucheon; \\ ${ }^{2}$ Department of Radiation Oncology, Samsung Medical Center, Sungkyunkwan University School of Medicine, Seoul; \\ ${ }^{3}$ Department of Radiation Oncology, Kyung Hee University Hospital at Gangdong, Seoul; \\ ${ }^{4}$ Department of Radiation Oncology, Korea Institute of Radiological \& Medical Sciences, Seoul; \\ ${ }^{5}$ Department of Social and Preventive Medicine, Sungkyunkwan University School of Medicine, Suwon, Korea
}

\begin{abstract}
Purpose: To determine the optimal radiotherapy technique for gastric mucosa-associated lymphoid tissue lymphoma (MALToma), we compared the dosimetric parameters and the risk of solid secondary cancer from scattered doses among anterior-posterior/ posterior-anterior parallel-opposed fields (AP/PA), anterior, posterior, right, and left lateral fields (4_field), 3-dimensional conformal radiotherapy (3D-CRT) using noncoplanar beams, and intensity-modulated radiotherapy composed of 7 coplanar beams (IMRT_co) and 7 coplanar and noncoplanar beams (IMRT_non).

Materials and Methods: We retrospectively generated 5 planning techniques for 5 patients with gastric MALToma. Homogeneity index ( $\mathrm{HI})$, conformity index $(\mathrm{Cl})$, and mean doses of the kidney and liver were calculated from the dose-volume histograms. Applied the Biological Effects of lonizing Radiation VII report to scattered doses, the lifetime attributable risk (LAR) was calculated to estimate the risk of solid secondary cancer.

Results: The best value of $\mathrm{Cl}$ was obtained with IMRT, although the $\mathrm{HI}$ varied among patients. The mean kidney dose was the highest with AP/PA, followed by 4_field, 3D-CRT, IMRT_Co, and IMRT_non. On the other hand, the mean liver dose was the highest with 4_field and the lowest with AP/PA. Compared with 4_field, the LAR for 3D-CRT decreased except the lungs, and the LAR for IMRT_co and IMRT_non increased except the lungs. However, the absolute differences were much lower than $<1 \%$.

Conclusion: Tailored RT techniques seem to be beneficial because it could achieve adjacent organ sparing with very small and clinically irrelevant increase of secondary solid cancer risk compared to the conventional techniques.
\end{abstract}

Keywords: Mucosa associated lymphoid tissue lymphoma, Radiotherapy, Secondary cancer risk, Stomach, Treatment planning

\section{Introduction}

Mucosa-associated lymphoid tissue lymphoma (MALToma) is a distinct clinical-pathologic entity and the stomach is the most common site of involvement $[1,2]$. In several studies, moderate-dose radiotherapy (RT) for gastric MALToma yielded

Received 5 September 2016, Revised 13 October 2016, Accepted 17 November 2016.

Correspondence: Do Hoon Lim, MD, PhD, Department of Radiation Oncology, Samsung Medical Center, Sungkyunkwan University School of Medicine, 81 Irwon-ro, Gangnam-gu, Seoul 06351, Korea. Tel: +82-2-3410-2603, Fax: +82-2-3410-2619, E-mail: dh8lim@skku.edu

(c) This is an Open Access article distributed under the terms of the Creative Commons Attribution Non-Commercial License (http://creativecommons.org/ licenses/by-nc/4.0/) which permits unrestricted non-commercial use, distribution, and reproduction in any medium, provided the original work is properly cited.

www.e-roj.org 
excellent outcomes, with the complete responses of $>95 \%$ [312]. Although RT for gastric MALToma is limited to the involved field, including the entire stomach and adjacent perigastric lymph nodes if these are involved, the organs at risk (OARs) such as the kidneys or liver are located near the stomach. Therefore, several planning techniques from anterior-posterior/ posterior-anterior fields (AP/PA) to 3-dimensional conformal radiotherapy (3D-CRT) have been used to reduce the radiation exposure to the OAR to be within tolerance limits. One study suggested that intensity-modulated radiotherapy (IMRT) showed the best dosimetric improvements to the kidney and liver, and it might be useful in selected patients [13]. However, IMRT generates higher scattered doses because of the longer beam-on time and increased head leakage and collimator scatter [14]. These factors would increase the risk of solid secondary cancer. Therefore, the optimal RT technique has yet not been well established.

In this study, we compared the dosimetric parameters and evaluated the risk of solid secondary cancer from scattered doses among AP/PA, 4_field, 3D-CRT using noncoplanar beams, IMRT composed of 7 coplanar beams (IMRT_co), and IMRT composed of 7 coplanar and noncoplanar beams (IMRT_non) to determine the optimal RT technique for gastric MALToma.

\section{Materials and Methods}

\section{Patients' selection and RT planning techniques}

Five patients with localized gastric MALToma, who were treated with RT at the Samsung Medical Center between 2012 and 2013, were included in this study. All patients underwent a simulation using 4-dimensional computed tomography (4D-CT). The 10 phases of the 4D-CT datasets were acquired by using a Real-time Position Management (RPM) system (Varian Medical System, Palo Alto, CA, USA). Using all of the CT datasets, the internal target volume (ITV) was defined as the sum total of the entire stomach at every respiratory stage. The

Table 1. Patients' characteristics and planning data

\begin{tabular}{|c|c|c|c|c|c|c|c|}
\hline No & Sex/age & Stage $^{a)}$ & Target volume & PTV (mL) & Planning technique & No. of beams & $M U^{b)}$ \\
\hline \multirow[t]{5}{*}{1} & $\mathrm{~F} / 49$ & $\|_{1}$ & Stomach + regional lymphatics & 1,441 & AP/PA & 2 & 215 \\
\hline & & & & & 4_Field & 4 & 234 \\
\hline & & & & & 3D-CRT & 5 (noncoplanar beam = 3) & 273 \\
\hline & & & & & IMRT_co & 7 & 1,441 \\
\hline & & & & & IMRT_non & 7 (noncoplanar beam = 2) & 1,383 \\
\hline \multirow[t]{5}{*}{2} & $\mathrm{~F} / 55$ & । & Stomach & 1,358 & $\mathrm{AP} / \mathrm{PA}$ & 2 & 219 \\
\hline & & & & & 4_Field & 4 & 233 \\
\hline & & & & & 3D-CRT & $4($ noncoplanar beam $=2)$ & 267 \\
\hline & & & & & IMRT_co & 7 & 1,371 \\
\hline & & & & & IMRT_non & 7 (noncoplanar beam = 2) & 1,373 \\
\hline \multirow[t]{5}{*}{3} & $\mathrm{M} / 50$ & $\|_{1}$ & Stomach + regional lymphatics & 1,589 & AP/PA & 2 & 222 \\
\hline & & & & & 4_Field & 4 & 239 \\
\hline & & & & & 3D-CRT & 6 (noncoplanar beam $=3$ ) & 279 \\
\hline & & & & & IMRT_co & 7 & 1,283 \\
\hline & & & & & IMRT_non & 7 (noncoplanar beam = 4) & 1,096 \\
\hline \multirow[t]{5}{*}{4} & $\mathrm{~F} / 53$ & I & Stomach & 1,043 & AP/PA & 2 & 214 \\
\hline & & & & & 4_Field & 4 & 223 \\
\hline & & & & & 3D-CRT & $4($ noncoplanar beam $=2)$ & 264 \\
\hline & & & & & IMRT_co & 7 & 1,200 \\
\hline & & & & & IMRT_non & $7($ noncoplanar beam $=3$ ) & 1,103 \\
\hline \multirow[t]{5}{*}{5} & $\mathrm{~F} / 58$ & $\|_{1}$ & Stomach + regional lymphatics & 1,246 & AP/PA & 2 & 204 \\
\hline & & & & & 4_Field & 4 & 223 \\
\hline & & & & & 3D-CRT & $4($ noncoplanar beam $=1)$ & 270 \\
\hline & & & & & IMRT_co & 7 & 1,512 \\
\hline & & & & & IMRT_non & 7 (noncoplanar beam = 2) & 1,370 \\
\hline
\end{tabular}

PTV, planning target volume; MU, monitor units; AP/PA, anterior-posterior/posterior-anterior fields; 3D-CRT, 3-dimensional conformal radiotherapy; IMRT_co, intensity-modulated radiotherapy composed of 7 coplanar beams; IMRT_non, IMRT composed of 7 coplanar and noncoplanar beams.

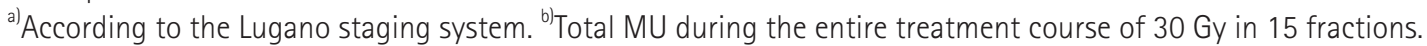


clinical target volume (CTV) was differently defined according to stage. The CTV for patients with stage I gastric MALToma was defined as the ITV plus a 1-cm margin in order to cover the perigastric lymph nodes. The CTV for patients with stage II 1 disease was defined as the CTV for stage I gastric MALToma plus a generous margin around the involved lymph nodes. The planning target volume (PTV) was defined as the CTV plus a $0.8-\mathrm{cm}$ margin. Normal tissues such as the right and left kidneys, liver, right and left lungs, and spinal cord were contoured on the planning CT image.
We sent the data into the Eclipse treatment planning system (Eclipse ver. 8.9.1; Varian Medical Systems) and retrospectively generated 5 planning techniques using a 10-MV photon: AP/PA parallel-opposed fields (AP/PA); anterior, posterior, right, and left lateral fields (4_field); 4-6 coplanar and noncoplanar fields (3D-CRT); IMRT_co; IMRT_non. The noncoplanar beams used for 3D-CRT and IMRT_non were mainly composed of inferior oblique beams to avoid the left kidney. Multi-leaf collimator apertures were created to encompass the PTV by $0.7 \mathrm{~cm}$ in all directions. The beam angles, weights, and dynamic wedges
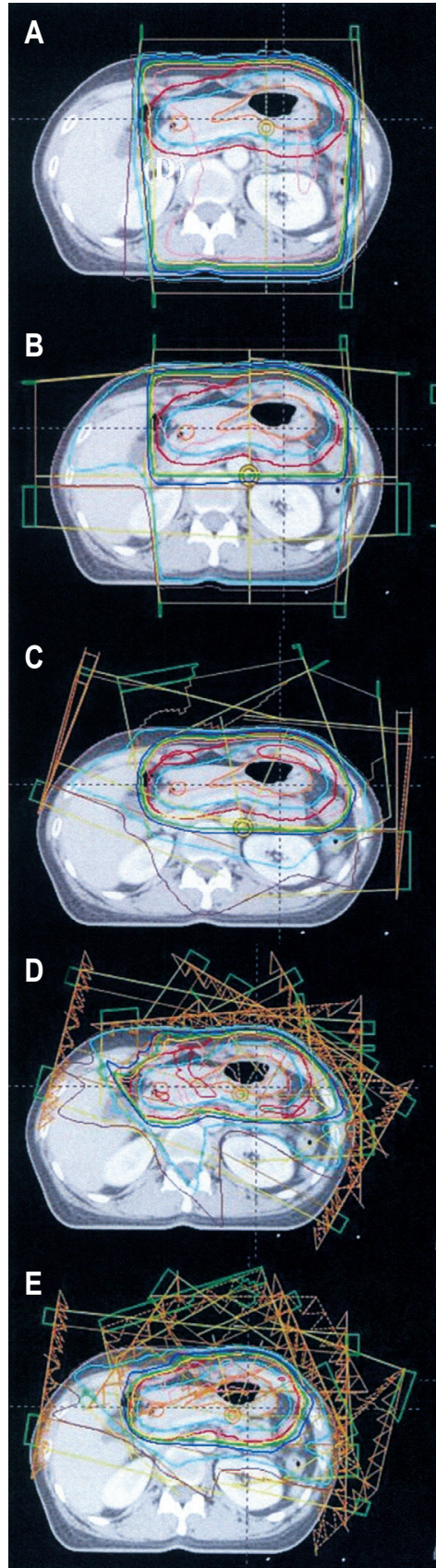
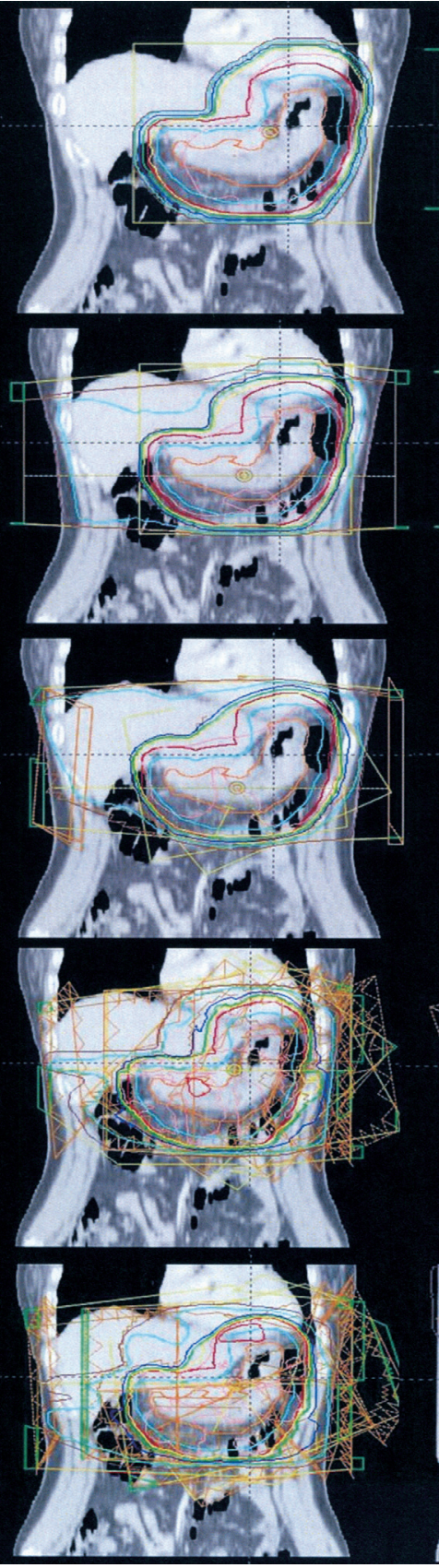
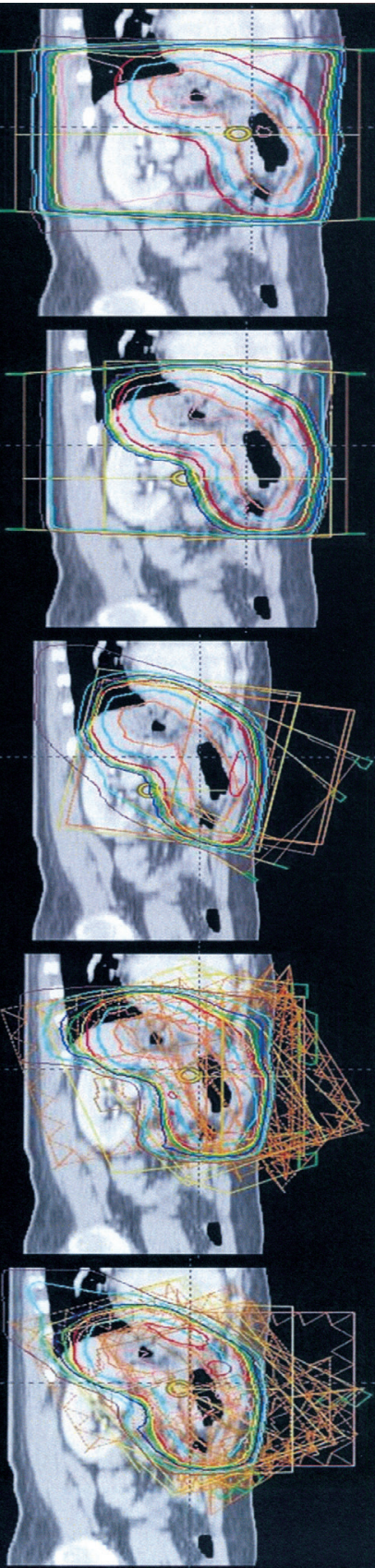

Fig. 1. Example of an actual treatment plan for patient \#4. (A) Anterior-posterior/ posterior-anterior fields; (B) anterior, posterior, right, and left lateral fields; (C) 3-dimensional conformal radiotherapy; (D) intensitymodulated radiotherapy (IMRT) composed of 7 coplanar beams; and (E) IMRT composed of 7 coplanar and noncoplanar beams. 
were optimized individually to achieve optimal PTV coverage, conformity, homogeneity, and dose limits of the OARs. In particular, doses to the left kidney and liver were reduced as much as possible without compromising the PTV coverage or conformity. The plans were normalized to the isocenter, and doses were prescribed at the isodose level that encompassed at least 95\% of the PTV, typically the 95\% isodose. All generated plans for each patient consisted of $30 \mathrm{~Gy}$ in 15 fractions. The patients' characteristics and planning data are described in detail in Table 1. A representative example of the treatment plan is presented in Fig. 1.

This study was approved by the Institutional Review Board of the Samsung Medical Center (2014-04-022). The review board waived the need for written informed consent from the participants because this study was retrospective nature and did not infringe on all patients' rights by anonymizing and de-identifying all patients' records and information prior to analysis.

\section{Dosimetric parameters for plan evaluation}

The homogeneity index (HI) is calculated as follows:

$$
H I=\left(\frac{D_{5}-D_{95}}{D_{p}}\right)
$$

where $D_{5}$ (the minimum doses in $5 \%$ of the PTV) and $D_{95}$ (the minimum doses in 95\% of the PTV) represent the minimum and maximum doses within the PTV and $D_{p}$ is the prescribed dose. A lower value indicates a more homogeneous dose distribution within the PTV; an ideal HI would be 0 [15].

The conformity index $(\mathrm{Cl})$ is calculated as follows:

$$
C l=\frac{B V_{95}}{P T V}
$$

where $\mathrm{BV}_{95}$ means the volume of the body receiving $95 \%$ of the prescribed dose. The $\mathrm{Cl}$ of 1 indicates an ideal conformation [16].

The mean doses in the kidneys and liver, and the percentage of liver volume that received at least $15 \mathrm{~Gy}\left(\mathrm{~V}_{15 \mathrm{~Gy}}\right)$ were calculated from the dose-volume histograms (DVH).

\section{Measurement of scattered doses}

For this study, we used a commercially available radiophotoluminescence glass dosimeter (RPLGD) (GD-302M; Asahi Techno Glass Co., Shizuoka, Japan) to measure the scattered doses. This RPLGD was rod-shaped with a diameter of $1.5 \mathrm{~mm}$ and a length of $8.5 \mathrm{~mm}$. The 13 RPLGDs were placed within the brain (right and left), thyroid (right and left), lungs (1 on right and 2 on left), rectum (right and left), bladder (right and left) and prostate/cervix (right and left) of a humanoid phantom (RANDO Phantom; The Phantom Laboratory, Salem, NY, USA). Fig. 2 shows the numbers and locations of the RPLGDs at each organ. A total of 25 planning techniques were delivered to the humanoid phantom by using the original planning parameters. The location of the isocenter was adjusted in the humanoid phantom until it was very similar to that of each patient. Radiation was delivered with RapidArc (Varian Medical Systems): one fraction of 2 Gy was delivered to the isocenter for each measurement. Next, the measured doses in the RPLGDs for the single fraction were multiplied by 15 to obtain the total scattered doses throughout the entire treatment course of $30 \mathrm{~Gy}$. Approximately, half of the total lung volume was included during each patient's CT simulation. Therefore, we estimated the scattered doses to the lungs by calculating the average of the absorbed doses according to the lung

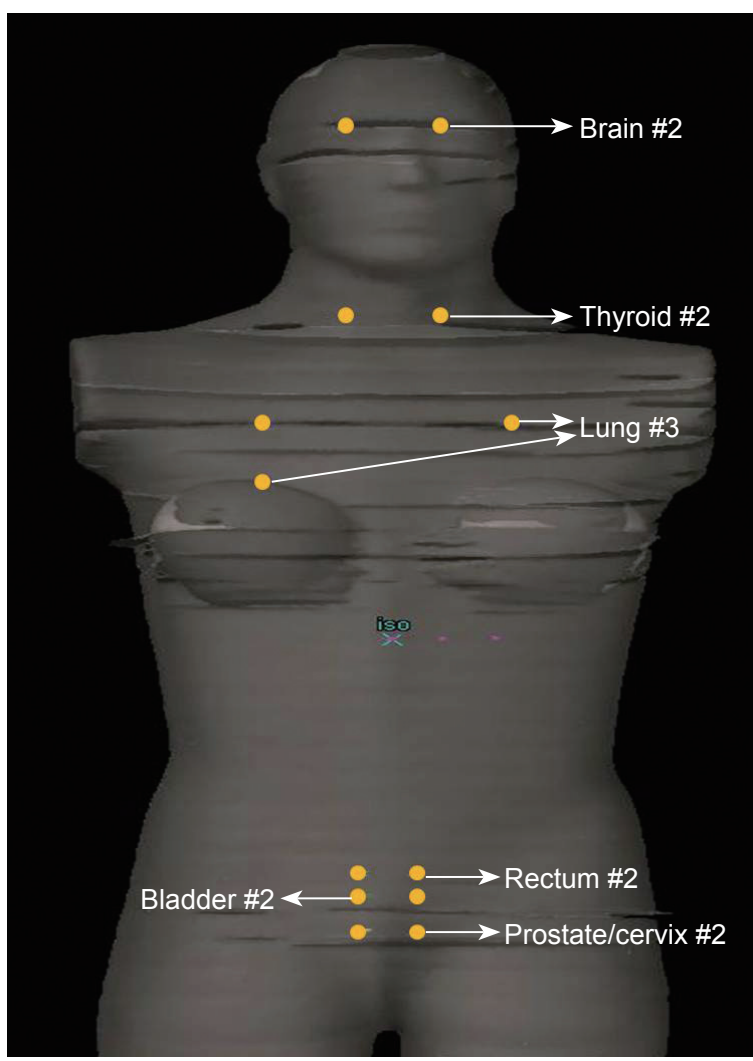

Fig. 2. The humanoid phantom equipped with radiophotoluminescence glass dosimeter. Yellow circle means the numbers and locations of the radio-photoluminescence glass dosimeters at the organs of interest. 
DVH from the original planning data and the measured doses from the RPLGDs located in the lung area of the humanoid phantom.

Finally, we adopted organ equivalent doses (OEDs) instead of the measured organ doses to assess the risk of solid secondary cancer from scattered doses, considering 3D dose distributions by 3D-CRT or IMRT [17]. The OED, which were based on a plateau dose-response model, was calculated as follows:

$$
O E D=\frac{1}{V} \sum_{i} V_{i}\left(\frac{1-\exp \left(-\delta D_{i}\right)}{\delta}\right)
$$

where $V$ is the whole volume, $V_{i}$ is a volume element, $D_{i}$ is the dose element, and $\delta$ is an organ-specific model parameter [18].

\section{Estimation of solid secondary cancer risk}

We used the Biological Effects of lonizing Radiation (BEIR) VII report and estimated the risk of solid secondary cancer in the organs of interest for male and female patients aged 40,50, 60,70 , and 80 years [19].

First, the excess absolute risk (EAR) and excess relative risk (ERR) were calculated as follows:

$\operatorname{EAR}(D, e, a) \operatorname{or} E R R(D, e, a)=\beta s D \exp \left(\gamma\left(\frac{\min (e-30)-30)}{10}\right)\right)\left(\frac{a}{60}\right) \eta$

where $D$ is the dose (OED), e is the age at exposure, $a$ is the attained age, and $\mathrm{s}$ is the sex. Sex-specific and organ-specific value of $\beta, \gamma$, and $\eta$ are obtained from Table $12-2$ in the BEIR VII report. The attained age was arbitrarily set at 20 years after exposure.

Next, we evaluated the lifetime attributable risk (LAR), which meant the solid cancer incidence per 100,000 exposed persons. The LAR for a person exposed to dose D (OED) at age e was calculated as follows:

$$
\operatorname{LAR}(D, \mathrm{e})=\int_{\mathrm{e}+L}^{100} M(D, \mathrm{e}, a) \times S(a) / s(\mathrm{e}) d a
$$

where the summation is from $a=e+L$ to 100 , a indicates the attained age, and $L$ is a risk-free latent period $(L=5$ years for solid cancer). The $M(D, e, a)$ is the EAR, S(a) is the probability of surviving until age $a$, and $S(a) / S(e)$ is the probability of surviving to age $a$, conditional on survival to exposed age e. The S(a)/S(e) values were obtained from a complete life-table 2012 for the Korea population [20]. And we compared the mean LAR of the 4 planning techniques in each organ with the mean LAR of 4_field, which most institutions currently use, to determine the relative differences.

\section{Statistical analysis}

The overall differences between the 5 planning techniques and the measured scattered doses in each organ were analyzed by using a generalized estimating equation method. A p-value of $<0.05$ was considered statistically significant. For organs with the statistically significant differences, the Tukey's multiple comparisons of the generalized estimating equation method were used to identify the differences between 4_field and the other 4 planning techniques. A p-value of $<0.05$ was considered significant. Statistical analyses were performed using SAS ver. 9.3 (SAS Institute Inc., Cary, NC, USA).

\section{Results}

\section{Dosimetric comparison}

Fig. $3 \mathrm{~A}$ shows the $\mathrm{HI}$ for each patient according to the 5 planning techniques. Although there was a trend toward a better $\mathrm{HI}$ with 4_field, the uniformity of dose distribution in the PTV varied according to the individual patient rather than the planning techniques. Fig. 3B shows the $\mathrm{Cl}$. The $\mathrm{Cl}$ for AP/PA ranged from 2-2.5, and this denoted a minor violation of the conformity. The $\mathrm{Cls}$ of the remaining 4 planning techniques ranged from 1-2, and they were considered to comply with the treatment plans, particularly in the case of IMRT.

The absorbed mean doses in the kidneys and liver, and $V_{15 G y}$ of the liver are indicated in Fig. 4. The mean kidney dose was the highest with AP/PA, followed by 4_field, 3D-CRT, IMRT_co, and IMRT_non. In contrast, the mean liver dose and $V_{15 G y}$ were the lowest with AP/PA and the highest with 4_field.

\section{The risk assessment of solid secondary cancer from scattered doses}

Table 2 summarizes the measured organ doses and OEDs from scattered doses throughout the entire treatment course of 30 Gy. The measured organ doses decreased as the distance from the PTV increased. In case of 3D-CRT, the noncoplanar beams increased the doses to organs above the PTV and decreased the doses to organs below the PTV than AP/PA and 4_field. Both IMRT_co and IMRT_non yielded the highest scattered doses, except in the lungs. IMRT_non used noncoplanar beams that increased the scattered doses to the lungs than IMRT_co despite using same dose-volume constraints as in IMRT_co. The OED at each organ was the same or slightly less relative to 
the measured doses by the parameter of $\delta$.

The LAR of each organ according to the age at exposure of 40-80 years, the sex, and the 5 planning techniques are indicated in Fig. 5. For the thyroid, there was no LAR data in a lack of the EAR data. The lungs represented the site with the most probable carcinogenic effects after RT, with estimated solid cancer incidence of 1.297-188.153 per 100,000 exposed males and 5.470-396.617 per 100,000 exposed females. The
A

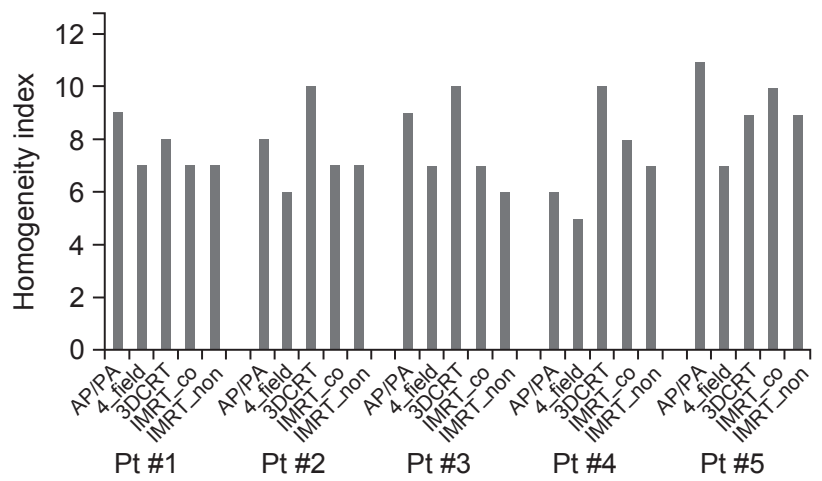

B

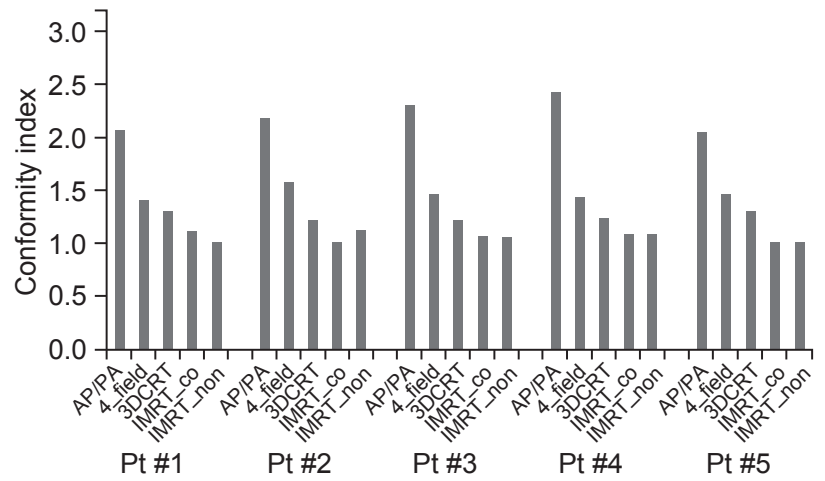

Fig. 3. (A) Homogeneity index and (B) conformity index for each patient according to the 5 planning techniques. AP/PA, anteriorposterior/posterior-anterior fields; 3D-CRT, 3-dimensional conformal radiotherapy; IMRT_co, intensity-modulated radiotherapy composed of 7 coplanar beams; IMRT_non, IMRT composed of 7 coplanar and noncoplanar beams.

A (Gy)

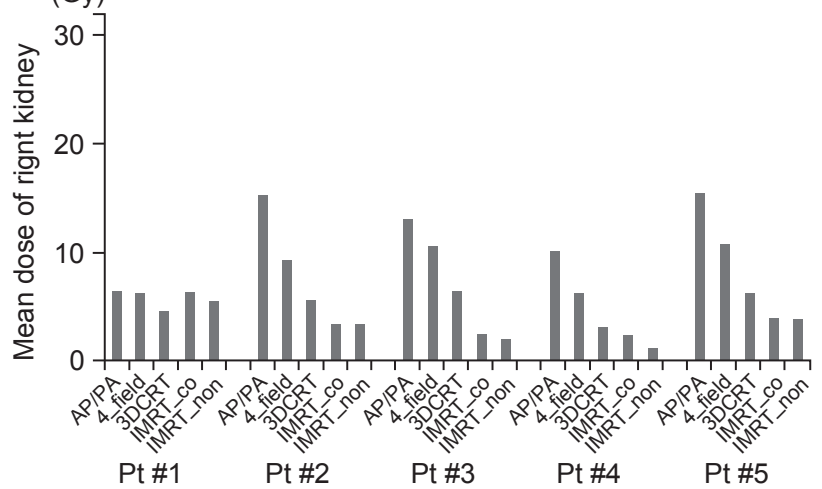

C (Gy)

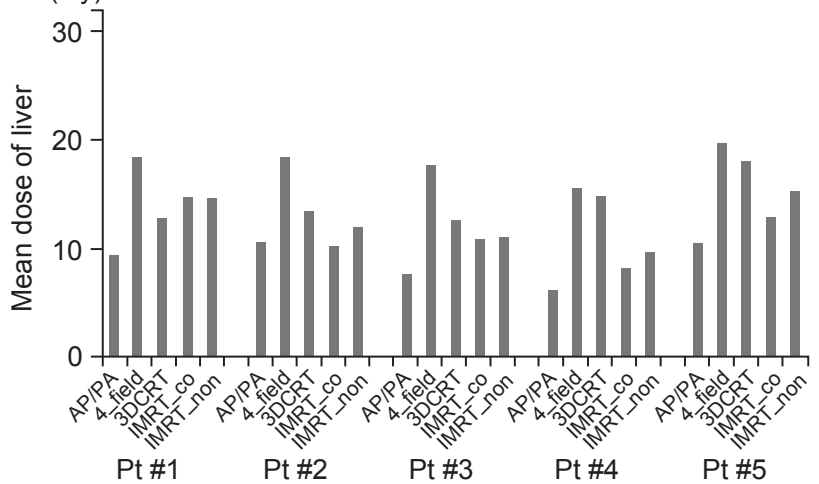

B (Gy)

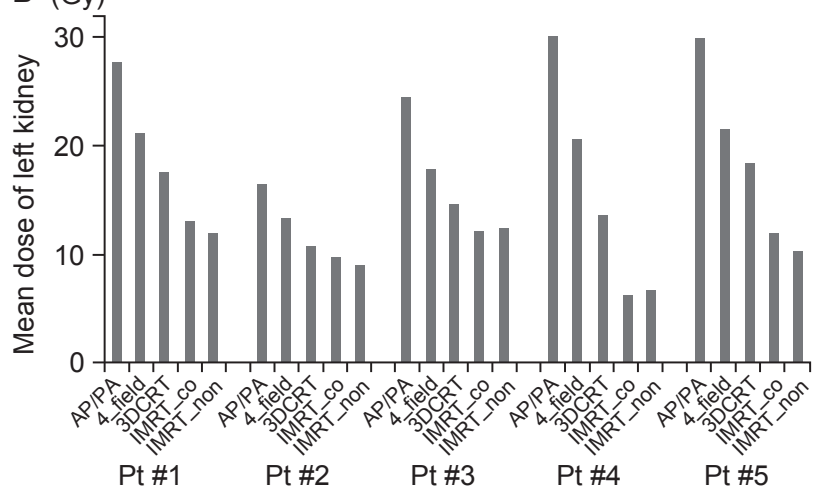

D $(\%)$

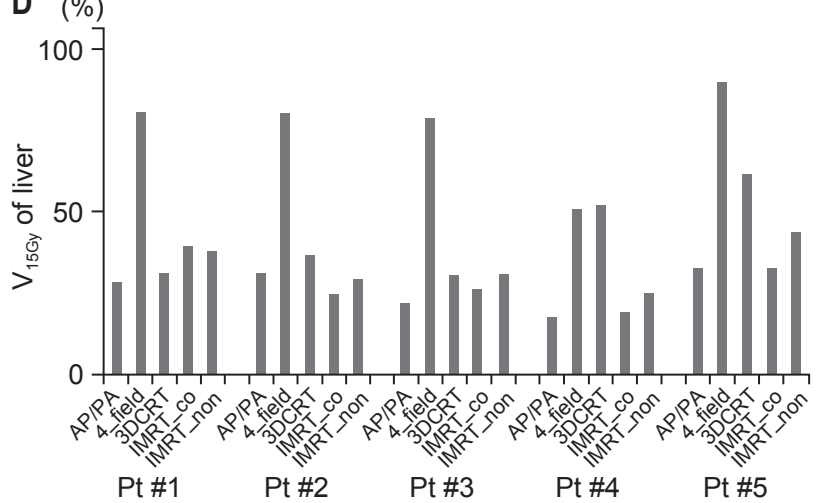

Fig. 4. The absorbed mean doses of the (A) right kidney, (B) left kidney, (C) liver, and (D) the percentage of liver volume receiving $\geq 15$ Gy (liver_ $V_{15 G y}$ ) for each patient according to the 5 planning techniques. AP/PA, anterior-posterior/posterior-anterior fields; 3D-CRT, 3-dimensional conformal radiotherapy; IMRT_co, intensity-modulated radiotherapy composed of 7 coplanar beams; IMRT_non, IMRT composed of 7 coplanar and noncoplanar beams. 
Table 2. The MOD and OED ${ }^{\text {a) }}$ from scattered doses throughout the entire treatment course of 30 Gy in 15 fractions for each patient

\begin{tabular}{|c|c|c|c|c|c|c|c|c|c|c|c|c|c|}
\hline \multirow{3}{*}{ Organ } & \multirow{3}{*}{$\begin{array}{l}\text { Planning } \\
\text { technique }\end{array}$} & \multicolumn{10}{|c|}{ Dose (Gy) } & \multicolumn{2}{|c|}{$p$-value } \\
\hline & & \multicolumn{2}{|c|}{ Patient \# 1} & \multicolumn{2}{|c|}{ Patient \#2 } & \multicolumn{2}{|c|}{ Patient \#3 } & \multicolumn{2}{|c|}{ Patient \#4 } & \multicolumn{2}{|c|}{ Patient \#5 } & \multirow{2}{*}{$\begin{array}{c}\text { Overall } \\
\text { comparison }^{\text {b) }}\end{array}$} & \multirow{2}{*}{$\begin{array}{c}\text { Multiple } \\
\text { comparison }\end{array}$} \\
\hline & & MOD & OED & MOD & OED & MOD & OED & MOD & OED & MOD & OED & & \\
\hline \multirow[t]{5}{*}{ Brain } & AP/PA & 0.022 & 0.022 & 0.013 & 0.013 & 0.025 & 0.025 & 0.027 & 0.027 & 0.034 & 0.034 & $<0.0001$ & 0.0757 \\
\hline & 4_Field & 0.024 & 0.024 & 0.033 & 0.033 & 0.041 & 0.040 & 0.031 & 0.031 & 0.036 & 0.036 & & - \\
\hline & 3D-CRT & 0.026 & 0.026 & 0.023 & 0.023 & 0.036 & 0.035 & 0.032 & 0.032 & 0.045 & 0.045 & & 0.9996 \\
\hline & IMRT_co & 0.069 & 0.068 & 0.053 & 0.053 & 0.053 & 0.053 & 0.063 & 0.063 & 0.067 & 0.067 & & $<0.0001$ \\
\hline & IMRT_non & 0.075 & 0.075 & 0.053 & 0.053 & 0.062 & 0.062 & 0.065 & 0.065 & 0.080 & 0.080 & & $<0.0001$ \\
\hline \multirow[t]{5}{*}{ Thyroid } & AP/PA & 0.068 & 0.067 & 0.060 & 0.059 & 0.076 & 0.074 & 0.078 & 0.076 & 0.088 & 0.086 & $<0.0001$ & 0.2102 \\
\hline & 4_Field & 0.065 & 0.064 & 0.095 & 0.092 & 0.109 & 0.105 & 0.097 & 0.094 & 0.084 & 0.082 & & - \\
\hline & 3D-CRT & 0.079 & 0.077 & 0.097 & 0.094 & 0.129 & 0.124 & 0.120 & 0.115 & 0.109 & 0.105 & & $<0.0001$ \\
\hline & IMRT_co & 0.170 & 0.160 & 0.151 & 0.143 & 0.175 & 0.164 & 0.174 & 0.164 & 0.174 & 0.164 & & $<0.0001$ \\
\hline & IMRT_non & 0.172 & 0.162 & 0.157 & 0.149 & 0.189 & 0.178 & 0.169 & 0.160 & 0.207 & 0.193 & & $<0.0001$ \\
\hline \multirow[t]{5}{*}{ Lung } & AP/PA & 2.999 & 2.415 & 2.785 & 2.276 & 2.447 & 2.048 & 3.083 & 2.468 & 2.352 & 1.982 & $<0.0001$ & $<0.0001$ \\
\hline & 4_Field & 3.963 & 2.988 & 3.626 & 2.797 & 3.275 & 2.587 & 3.759 & 2.873 & 3.128 & 2.497 & & - \\
\hline & 3D-CRT & 4.434 & 3.238 & 4.388 & 3.215 & 3.895 & 2.950 & 4.498 & 3.271 & 3.806 & 2.900 & & $<0.0001$ \\
\hline & IMRT_co & 3.384 & 2.654 & 2.828 & 2.305 & 2.578 & 2.138 & 2.984 & 2.406 & 2.549 & 2.118 & & $<0.0001$ \\
\hline & IMRT_non & 4.017 & 3.017 & 3.018 & 2.427 & 3.372 & 2.646 & 3.817 & 2.906 & 2.995 & 2.413 & & 0.8955 \\
\hline \multirow[t]{5}{*}{ Rectum } & AP/PA & 0.161 & 0.158 & 0.168 & 0.164 & 0.203 & 0.198 & 0.186 & 0.182 & 0.233 & 0.226 & $<0.0001$ & 0.0014 \\
\hline & 4_Field & 0.162 & 0.159 & 0.119 & 0.117 & 0.129 & 0.127 & 0.104 & 0.103 & 0.190 & 0.185 & & - \\
\hline & 3D-CRT & 0.105 & 0.104 & 0.093 & 0.092 & 0.091 & 0.090 & 0.070 & 0.069 & 0.153 & 0.150 & & $<0.0001$ \\
\hline & IMRT_co & 0.284 & 0.274 & 0.246 & 0.238 & 0.279 & 0.269 & 0.265 & 0.256 & 0.328 & 0.314 & & $<0.0001$ \\
\hline & IMRT_non & 0.287 & 0.276 & 0.259 & 0.250 & 0.240 & 0.233 & 0.232 & 0.225 & 0.287 & 0.277 & & $<0.0001$ \\
\hline \multirow[t]{5}{*}{ Bladder } & AP/PA & 0.118 & 0.089 & 0.128 & 0.094 & 0.147 & 0.104 & 0.139 & 0.099 & 0.162 & 0.110 & $<0.0001$ & 0.0046 \\
\hline & 4_Field & 0.121 & 0.090 & 0.090 & 0.072 & 0.106 & 0.082 & 0.085 & 0.069 & 0.140 & 0.100 & & - \\
\hline & 3D-CRT & 0.099 & 0.078 & 0.080 & 0.066 & 0.085 & 0.069 & 0.063 & 0.054 & 0.129 & 0.095 & & $<0.0001$ \\
\hline & IMRT_co & 0.260 & 0.144 & 0.232 & 0.136 & 0.270 & 0.147 & 0.241 & 0.139 & 0.287 & 0.151 & & $<0.0001$ \\
\hline & IMRT_non & 0.259 & 0.144 & 0.231 & 0.136 & 0.235 & 0.137 & 0.236 & 0.137 & 0.296 & 0.153 & & $<0.0001$ \\
\hline \multirow{5}{*}{$\begin{array}{l}\text { Prostate/ } \\
\text { cervix }\end{array}$} & AP/PA & 0.090 & 0.087 & 0.096 & 0.092 & 0.126 & 0.121 & 0.119 & 0.114 & 0.123 & 0.118 & $<0.0001$ & 0.0132 \\
\hline & 4_Field & 0.093 & 0.090 & 0.070 & 0.069 & 0.090 & 0.087 & 0.065 & 0.064 & 0.103 & 0.099 & & - \\
\hline & 3D-CRT & 0.085 & 0.082 & 0.061 & 0.059 & 0.062 & 0.061 & 0.052 & 0.051 & 0.110 & 0.106 & & 0.2482 \\
\hline & IMRT_co & 0.190 & 0.178 & 0.176 & 0.165 & 0.199 & 0.185 & 0.175 & 0.165 & 0.217 & 0.201 & & $<0.0001$ \\
\hline & IMRT_non & 0.191 & 0.178 & 0.180 & 0.169 & 0.167 & 0.157 & 0.184 & 0.172 & 0.204 & 0.190 & & $<0.0001$ \\
\hline
\end{tabular}

MOD, measured organ dose; OED, organ equivalent dose; AP/PA, anterior-posterior/posterior-anterior fields; 3D-CRT, 3-dimensional conformal radiotherapy; IMRT_co, intensity-modulated radiotherapy composed of 7 coplanar beams; IMRT_non, IMRT composed of 7 coplanar and noncoplanar beams.

${ }^{a}$ OED was calculated based on a plateau dose-response model.

${ }^{b)}$ The overall difference between the 5 planning techniques and MOD was analyzed using a generalized estimating equation method.

c'Tukey's multiple comparisons of generalized estimating equation method was used to identify the difference between 4_field and the other 4 planning technique.

incidence of solid secondary cancer was age-dependent, and increased as the age at exposure decreased. In terms of the sex, there was variability. Younger male had higher LAR in the rectum and bladder than female. With an increasing age at exposure, however, older male had lower LAR than female because female live longer and have a higher probability of survival. Relative to 4_field in terms of planning techniques, the mean LAR for AP/PA decreased by 19\%-26\% in the brain and lungs but increased by $20 \%-34 \%$ in the other organs, whereas the mean LAR for 3D-CRT increased by $13 \%$ in the lungs but decreased by $27 \%$ in the other organs. The mean LAR for IMRT_co relatively decreased by 15\% in the lungs but increased by $73 \%-126 \%$ in the other organs, whereas the mean LAR for IMRT_non decreased by only 2\% in the lungs and increased by $71 \%-119 \%$ in the other organs. The greatest difference in the mean LAR was observed between 4_field and IMRT in the cervix, and the estimated solid cancer incidence rates per 100,000 exposed females were 10.524 after 4 
A

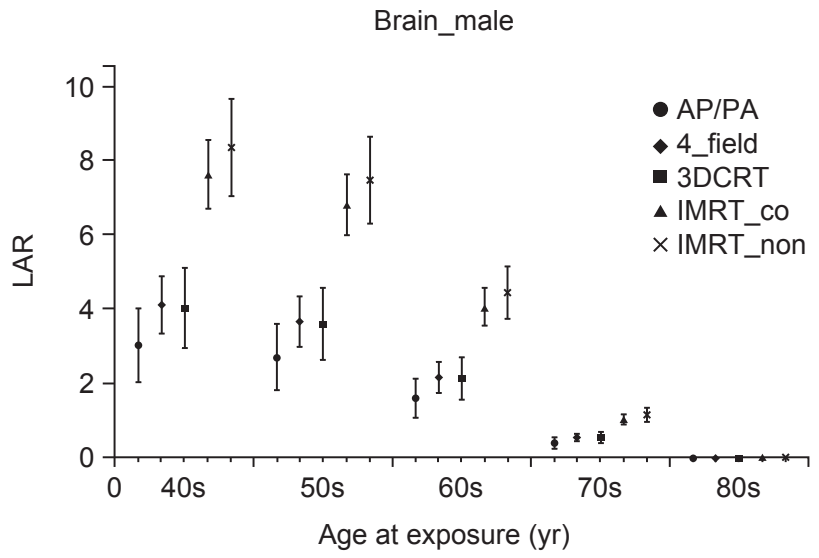

C

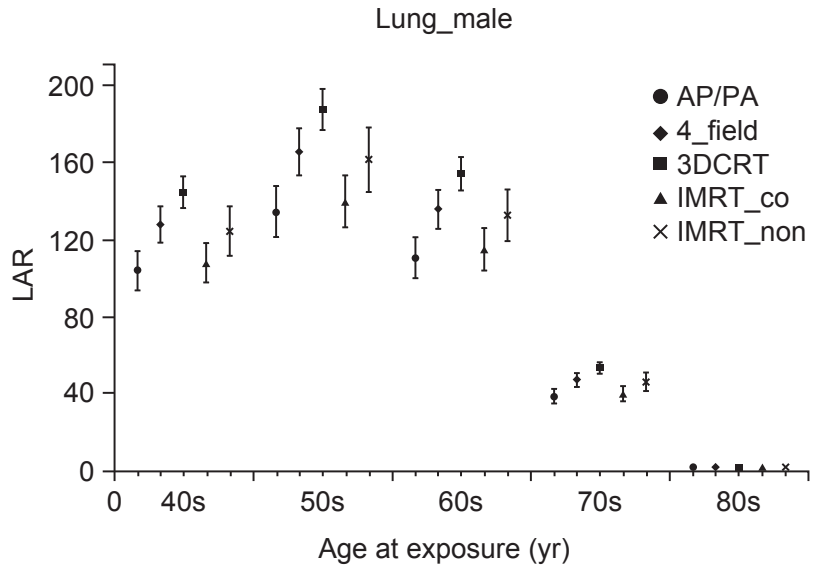

$E$

Rectum_male

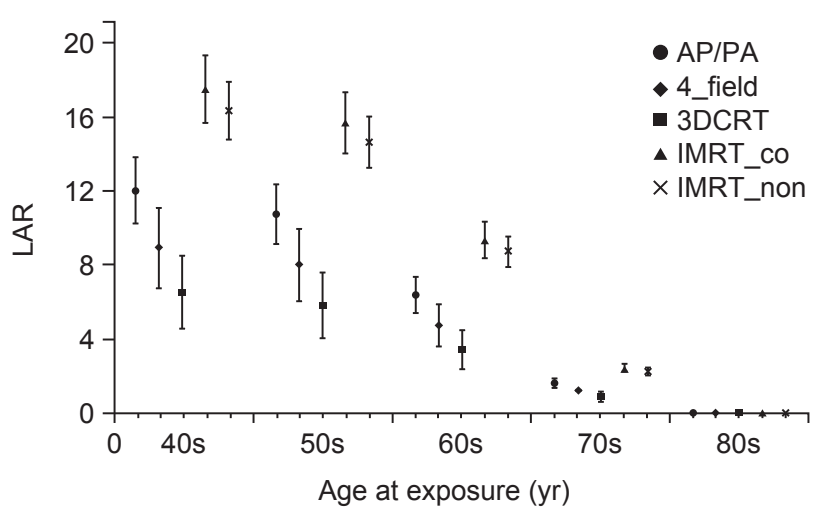

B

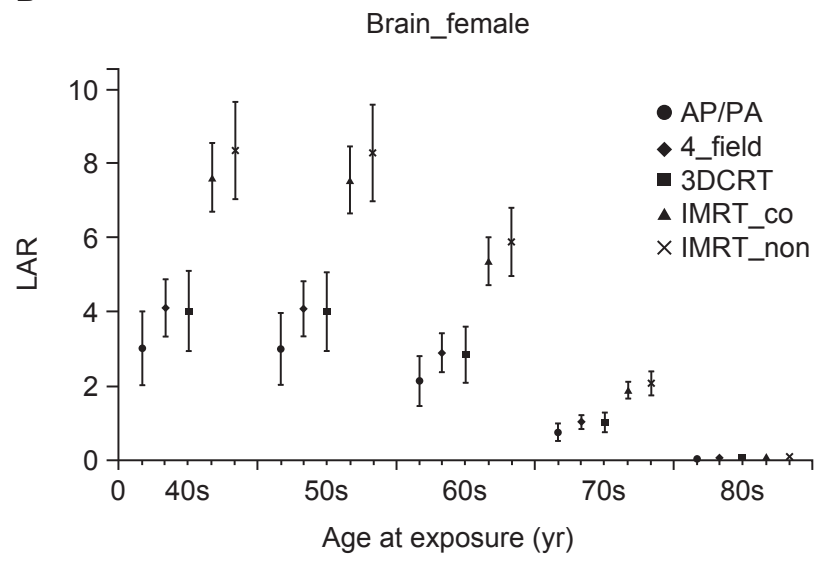

D

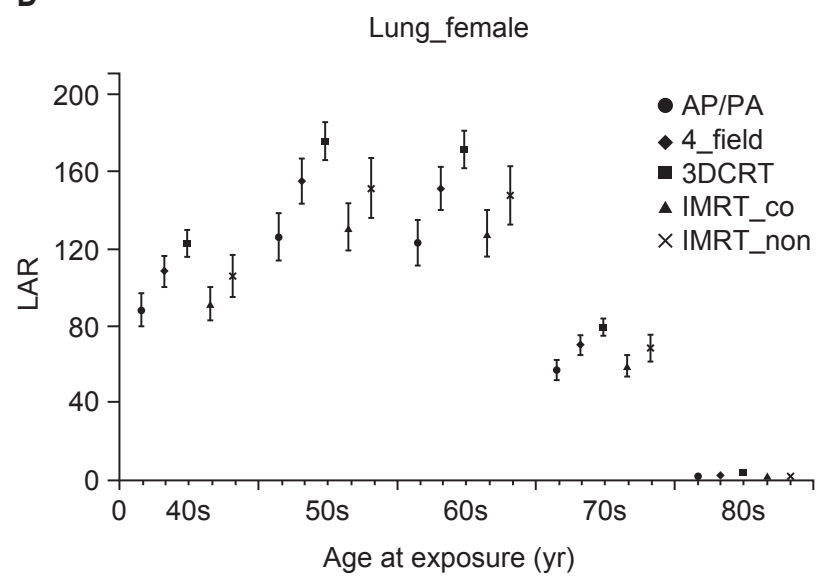

$\mathbf{F}$

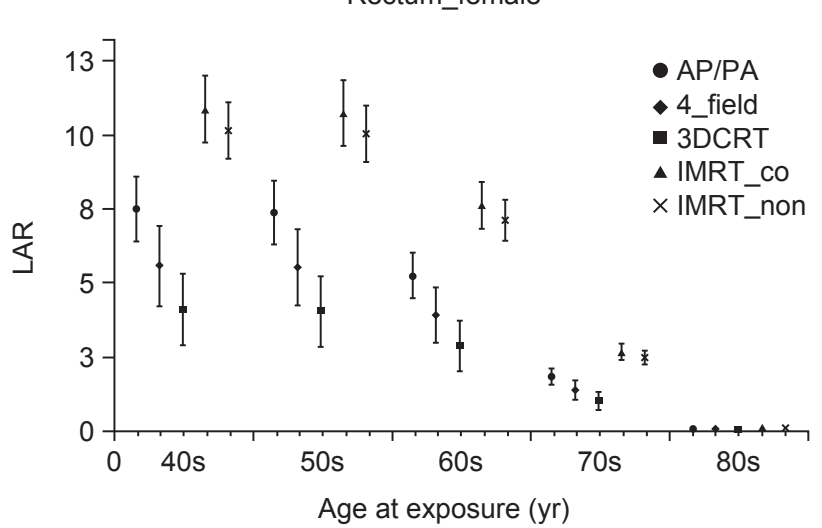

Fig. 5. Lifetime attributable risk ( $L A R$, the solid cancer incidence per 100,000 exposed persons) for each organ according to the age at exposure of 40-80 years, the sex, and the 5 planning techniques (mean \pm standard deviation). (A) Brain_male, (B) brain_female, (C) lung_male, (D) lung_female, (E) rectum_male, (F) rectum_female, (G) bladder_male, (H) bladder_female, (I) postate_male, and (J) cervix_female. AP/PA, anterior-posterior/posterior-anterior fields; 3D-CRT, 3-dimensional conformal radiotherapy; IMRT_co, intensitymodulated radiotherapy composed of 7 coplanar beams; IMRT_non, IMRT composed of 7 coplanar and noncoplanar beams. 
G

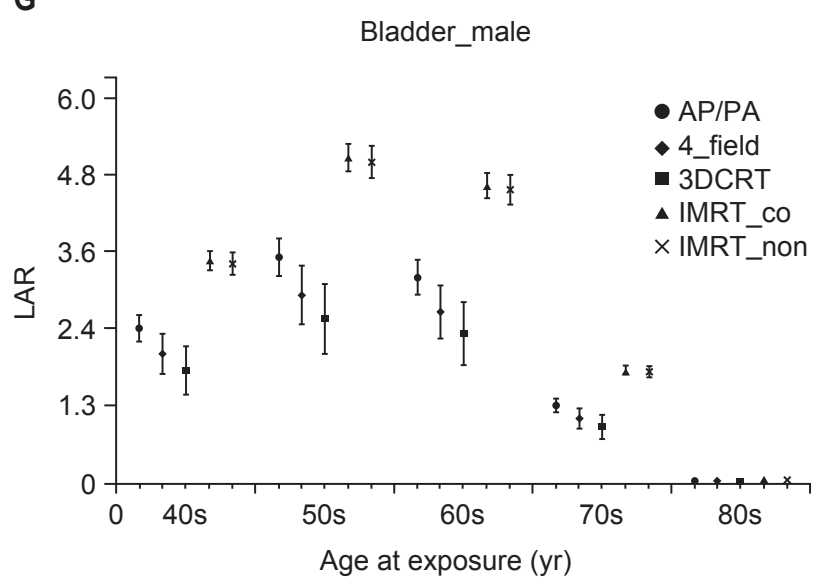

I

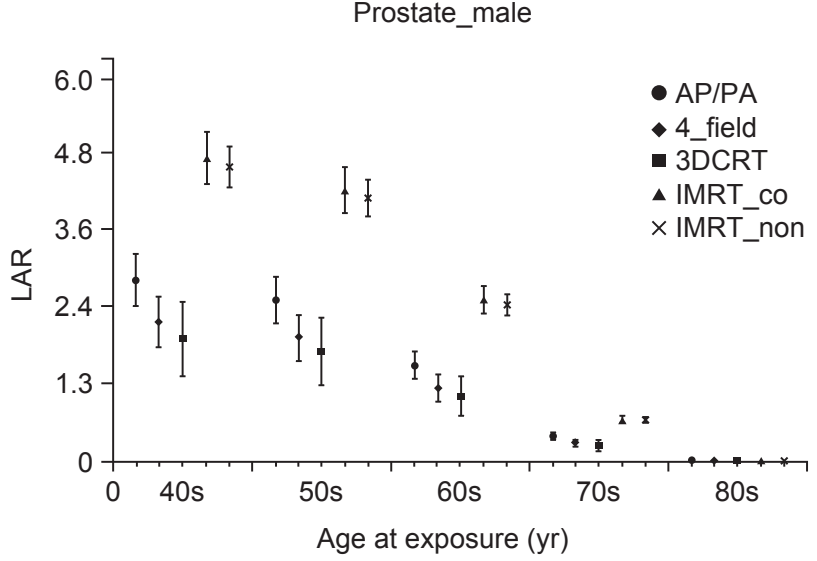

Fig. 5. Continued.

field, 23.764 after IMRT_co, and 23.004 after IMRT_non. The difference of absolute value, however, was much lower than $1 \%$.

\section{Discussion and Conclusion}

To our knowledge, this was the first study to evaluate the optimal planning technique using modern RT techniques for gastric MALToma. When we compared the dosimetric parameters of the 5 planning techniques, IMRT yielded the best value for conformity, and followed by 3D-CRT, 4_field, and AP/PA. In terms of normal tissue sparing, the mean kidney dose was the highest with AP/PA, followed by 4_field, 3D-CRT, IMRT_CO, and IMRT_non. The risk of renal toxicity after RT for gastric MALToma increased along with the increase of irradiated dose to the kidney [21]. Therefore, most institutions use the 3_field, 4_field or multi-fields technique rather than AP/PA to reduce the dose to the kidney with no reported renal
H
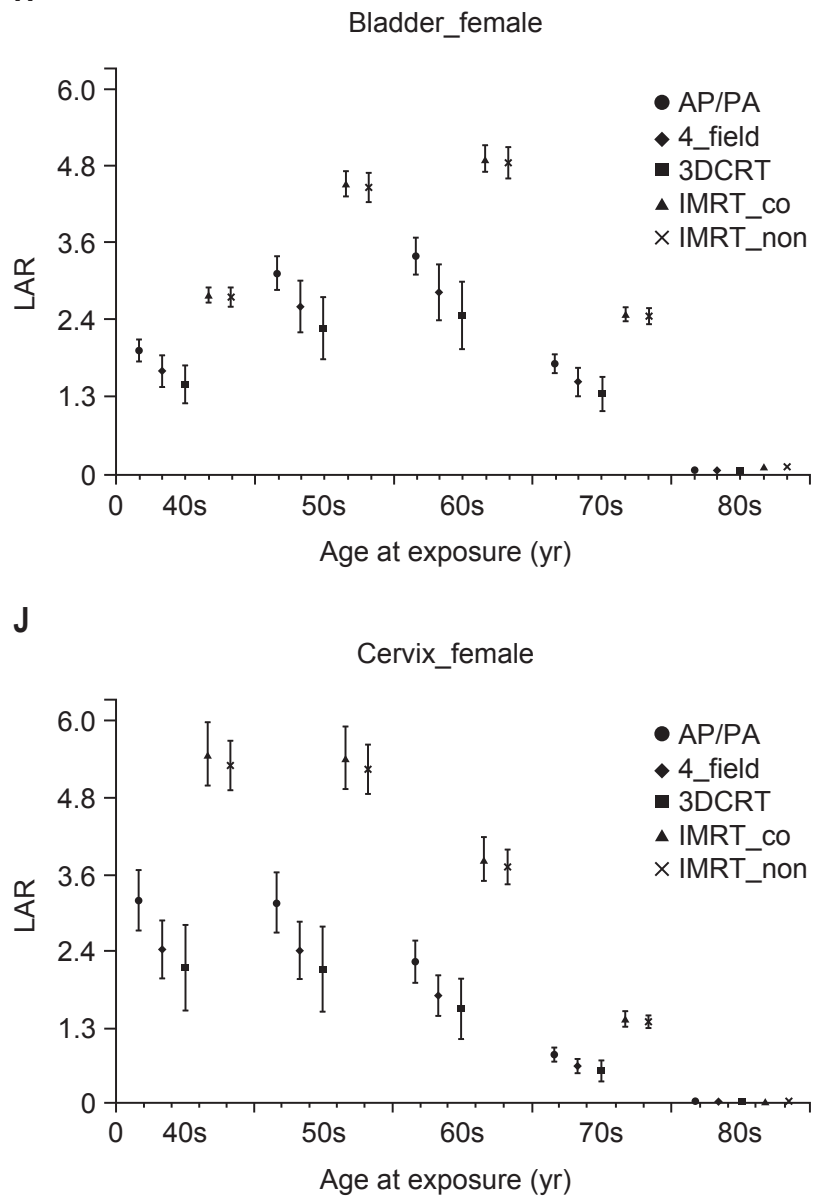

toxicity. However, the use of these techniques leads to an increase the dose to the liver than AP/PA, and increases the probability of hepatic toxicity. Tanaka [22] reported hepatic dysfunction of 70\% after RT using 3_field or 4_field to gastric lymphoma. For patients with $\mathrm{V}_{10 G \mathrm{y}}>60 \%, \mathrm{~V}_{15 \mathrm{~Gy}}>50 \%$ or $V_{206 y}>30 \%$ of the liver, the incidence of hepatic dysfunction increased significantly. They improved 2-50 months after RT, but 6 patients experienced persistently increased alkaline phosphatase levels within the follow-up period. If a patient has comorbidities unrelated to gastric lymphoma, the occurrence and prolongation of hepatic toxicity might disturb further treatment. Therefore, authors suggested that the dose to the liver should remain as low as possible. Some studies about the liver sparing effect between 3D-CRT and IMRT for hepatocellular carcinoma reported that the high dose region was significantly smaller in IMRT, although the low dose region was significantly smaller in 3D-CRT $[23,24]$. These support that IMRT would have a role in the liver protection by reducing 
Table 3. Incidence of secondary cancer from previous studies using RT for gastric MALToma

\begin{tabular}{|c|c|c|c|c|c|c|c|}
\hline Study & $\begin{array}{l}\text { No. of } \\
\text { patients }\end{array}$ & $\begin{array}{l}\text { Treatment } \\
\text { period }\end{array}$ & $\begin{array}{c}\text { RT } \\
\text { technique }\end{array}$ & $\begin{array}{l}\text { Median RT dose } \\
(\text { Gy)/fraction }\end{array}$ & $\begin{array}{l}\text { Median } \\
\text { F/U (yr) }\end{array}$ & $\begin{array}{l}\text { Incidence of } \\
\text { secondary } \\
\text { cancer }(\%)\end{array}$ & Details \\
\hline Lin et al. [5] & 18 & 1980-2003 & $\begin{array}{l}\text { AP/PA or } \\
\text { multiple } \\
\text { fields }\end{array}$ & $\begin{array}{l}30 / 20 \\
\text { (range, 30-36) }\end{array}$ & 4.5 & 17 & $\begin{array}{l}\text { In_field: gastric cancer at } 24 \\
\text { months }(n=1) \\
\text { In_field: hepatoma at } 36 \text { months } \\
(n=1) \\
\text { Out_field: bladder cancer at } \\
42 \text { months }(n=1)\end{array}$ \\
\hline \multirow[t]{2}{*}{ Goda et al. [8] } & $\begin{array}{l}167^{\mathrm{a})} \\
(25)^{\mathrm{b})}\end{array}$ & 1989-2004 & $\begin{array}{l}\text { Simple } \\
\text { beam or } \\
\text { 3D-CRT }\end{array}$ & $\begin{array}{l}25 / 10 \text { for } \\
\text { orbital } \\
\text { MALToma } \\
\text { (range, 25-35) }\end{array}$ & 7.4 & 11 & $\begin{array}{l}\text { In_field at } 5 \text { years and } 7 \text { years } \\
(n=2) \\
\text { Others were not specified. }\end{array}$ \\
\hline & & & & $\begin{array}{l}30 \text { for non-or- } \\
\text { bital MALToma } \\
\text { (range, 17.5-35) }\end{array}$ & & & $\begin{array}{l}\text { Most of secondary malignancy } \\
\text { were at distant sited from the } \\
\text { irradiated area, and were of } \\
\text { diverse histology. }\end{array}$ \\
\hline Okada et al. [9] & 22 & 1997-2011 & $\begin{array}{l}\text { 2D } \\
\text { technique }\end{array}$ & $30 / 20$ & 6 & 5 & $\begin{array}{l}\text { Out_field: lung cancer at } 3 \text { years } \\
(n=1)\end{array}$ \\
\hline Wirth et al. [10] & 102 & 1981-2004 & $\begin{array}{l}\text { AP_PA or } \\
\text { multiple } \\
\text { fields }\end{array}$ & $\begin{array}{l}\text { 40/22 } \\
\text { (range, 26-46) }\end{array}$ & 7.9 & 13 & $\begin{array}{l}\text { When stomach and regional LNs } \\
\text { were included, } \\
\text { In_field: colon cancer }(n=2) \\
\text { Out_field: bladder cancer }(n=1) \\
\text { Uncertain relationship: } 1 \text { breast } \\
\text { cancer and } 1 \text { lung cancer }(n=2) \\
\text { When whole abdomen was } \\
\text { included, } \\
\text { In_field: } 2 \text { prostate cancer and } 1 \\
\text { endometrial cancer }(n=3) \\
\text { Out_field: } 1 \text { larynx cancer and } 1 \\
\text { neck skin cancer }(n=2) \\
\text { Uncertain relationship: } 1 \text { breast } \\
\text { cancer, } 1 \text { lung cancer, and } 1 \text { skin } \\
\text { cancer }(n=3)\end{array}$ \\
\hline
\end{tabular}

RT, radiotherapy; MALToma, mucosa-associated lymphoid tissue lymphoma; F/U, follow-up; AP/PA, anterior-posterior/posterior-anterior fields; 3D-CRT, 3-dimensional conformal radiotherapy; 2D, 2-dimensional.

${ }^{a}$ Presenting sites were as follows: orbital adnexa in 71 patients, salivary glands in 28 patients, stomach in 25 patients, thyroid in 21 patients, and other sites in 22 patients; ${ }^{b}$ stomach only.

the high dose region. In our study, $V_{156 y}$ of the liver $>50 \%$ was observed for all patients with 4_field and for 2 patients with 3D-CRT. In this clinical setting, IMRT planning technique should be considered to reduce hepatic toxicity. However, there was no significant difference between IMRT_co and IMRT_ non. Therefore, we recommend IMRT_co rather than IMRT_ non because of the reduced treatment time required for couch rotation and the reduced mechanical load on the treatment machine.

Because patients with gastric MALToma tend to have a prolonged survival, the development of secondary cancer is an important issue. The influence of RT to secondary cancer for gastric MALToma, however, is controversial. Although RT is considered as a major risk factor for the development of secondary cancers, only $8 \%$ of secondary cancers are duo to RT and the remainders are due to genetic factors, lifestyle behaviors, and aging [25]. Some studies that followed patients for $>7$ years after RT to gastric MALToma reported variable secondary cancer incidence of $5-17 \%$, as shown in Table 3 [5,8-10]. One study demonstrated that most of solid secondary cancers developed at sites distant from the irradiated area [8]. In this study, we applied 3D-CRT and IMRT to maximize normal 
tissue sparing. However, the noncoplanar beams of 3D-CRT and IMRT_non increase the volumes of normal tissues exposed to lower RT doses because of the longer beam pathway. IMRT has more monitor units and induces a larger total body dose from leakage $[14,26]$. These increases of scattered dose might increase the risk of solid secondary cancer, especially at sites distant from the irradiated area. When we estimated the risk of solid secondary cancer by using the BEIR VII report, the LAR decreased as the age at exposure increased. The mean LAR for 3D-CRT increases by $13 \%$ in the lungs but decreases by $0 \%-27 \%$ in the other organs than 4_field, which is currently used in most institutions. On the other hand, IMRT relatively increase the LAR approximately 2-fold than 4_field in all organs except the lungs. The difference of absolute value between IMRT and 4_field, however, was much lower than 1\% in all organs. Therefore, 3D-CRT could be safely applied to treat gastric MALToma without increasing the risk of solid secondary cancer. If a 3D-CRT-based planning technique shows $V_{15 G y}$ of the liver $>50 \%$, IMRT_co would be considered to minimize hepatic toxicity, especially for patients with underlying liver disease because the absolute incidence of solid secondary cancer associated with IMRT is very small.

There were following limitations in the current study. First, we used the BEIR VII report to estimate the risk of solid secondary cancer. However, the risk estimation model contained inherent uncertainty with respect to the epidemiological data and our understanding of exactly how radiation exposure increases the risk of cancer, although the BEIR VII report provided the organ, sex, and age-specific model parameters [19]. These statistical uncertainties and assumptions have introduced considerable uncertainty. Second, we measured scattered doses by using a humanoid phantom. The phantom did not account for inter-patient anatomical variations in the organs of interest. These variations might induce changes in the measured organ doses and substantial changes in the LAR. Therefore, we need to conduct long-term follow-up in a clinical setting.

In conclusion, all planning techniques provided appropriate PTV coverage and conformity for gastric MALToma, and both IMRT techniques offered the greatest dosimetric benefit. In terms of normal tissue sparing, the mean dose to the kidney was the highest with AP/PA, followed by 4_field, 3D-CRT, IMRT_co, and IMRT_non. On the other hand, the mean liver dose and $V_{15 G y}$ were the lowest with AP/PA and the highest with 4_field. In terms of the risk of solid secondary cancer, 3D-CRT did not increase the LAR when compared with AP/ PA and 4_field. On the other hand, IMRT increased the LAR by approximately 2-fold in all organs except for the lungs, relatively. However, the absolute differences between the planning techniques were very small. Therefore, tailored RT techniques seem to be beneficial because it could achieve adjacent organ sparing with very small and clinically irrelevant increase of secondary solid cancer risk compared to the conventional techniques.

\section{Conflict of Interest}

No potential conflict of interest relevant to this article was reported.

\section{Acknowledgments}

This work was supported by the Soonchunhyang University Research Fund.

\section{References}

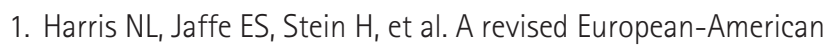
classification of lymphoid neoplasms: a proposal from the International Lymphoma Study Group. Blood 1994;84:136192.

2. Harris NL, Jaffe ES, Diebold J, et al. World Health Organization classification of neoplastic diseases of the hematopoietic and Iymphoid tissues: report of the Clinical Advisory Committee meeting-Airlie House, Virginia, November 1997. J Clin Oncol 1999;17:3835-49.

3. Tsang RW, Gospodarowicz MK, Pintilie M, et al. Localized mucosa-associated lymphoid tissue lymphoma treated with radiation therapy has excellent clinical outcome. J Clin Oncol 2003;21:4157-64.

4. Koch $P$, Probst $A$, Berdel WE, et al. Treatment results in localized primary gastric Iymphoma: data of patients registered within the German multicenter study (GIT NHL 02/96). J Clin Oncol 2005;23:7050-9.

5. Lin $M L$, Wirth $A$, Chao $M$, et al. Radiotherapy for low-grade gastric marginal zone lymphoma: a retrospective study. Intern Med J 2007;37:172-80.

6. Vrieling $C$, de Jong $D$, Boot $H_{1}$, de Boer JP, Wegman $F$, Aleman $B M$. Long-term results of stomach-conserving therapy in gastric MALT Iymphoma. Radiother Oncol 2008;87:405-11.

7. Gobbi PG, Corbella F, Valentino F, et al. Complete long-term response to radiotherapy of gastric early-stage marginal zone lymphoma resistant to both anti-Helicobacter pylori antibiotics and chemotherapy. Ann Oncol 2009;20:465-8.

8. Goda JS, Gospodarowicz M, Pintilie M, et al. Long-term outcome in localized extranodal mucosa-associated lymphoid 
tissue Iymphomas treated with radiotherapy. Cancer 2010;116:3815-24.

9. Okada $H$, Takemoto $M$, Kawahara $Y$, et al. A prospective analysis of efficacy and long-term outcome of radiation therapy for gastric mucosa-associated Iymphoid tissue lymphoma. Digestion 2012;86:179-86.

10. Wirth A, Gospodarowicz M, Aleman BM, et al. Long-term outcome for gastric marginal zone lymphoma treated with radiotherapy: a retrospective, multi-centre, International Extranodal Lymphoma Study Group study. Ann Oncol 2013;24:1344-51.

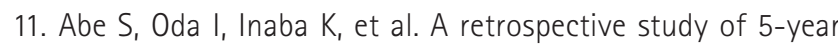
outcomes of radiotherapy for gastric mucosa-associated Iymphoid tissue lymphoma refractory to Helicobacter pylori eradication therapy. Jpn J Clin Oncol 2013;43:917-22.

12. Kim SW, Lim DH, Ahn YC, et al. Clinical outcomes of radiation therapy for early-stage gastric mucosa-associated lymphoid tissue lymphoma. World J Gastroenterol 2013;19:6062-8.

13. Della Biancia C, Hunt M, Furhang E, Wu E, Yahalom J. Radiation treatment planning techniques for lymphoma of the stomach. Int J Radiat Oncol Biol Phys 2005;62:745-51.

14. Ruben JD, Lancaster $C M$, Jones $P$, Smith RL. A comparison of out-of-field dose and its constituent components for intensity-modulated radiation therapy versus conformal radiation therapy: implications for carcinogenesis. Int J Radiat Oncol Biol Phys 2011:81:1458-64.

15. Kataria T, Sharma K, Subramani V, Karrthick KP, Bisht SS. Homogeneity index: an objective tool for assessment of conformal radiation treatments. J Med Phys 2012;37:207-13.

16. Feuvret L, Noel G, Mazeron JJ, Bey P. Conformity index: a review. Int J Radiat Oncol Biol Phys 2006;64:333-42.

17. Schneider U, Zwahlen D, Ross D, Kaser-Hotz B. Estimation of radiation-induced cancer from three-dimensional dose distributions: concept of organ equivalent dose. Int J Radiat Oncol Biol Phys 2005;61:1510-5.

18. Schneider U, Kaser-Hotz B. Radiation risk estimates after radiotherapy: application of the organ equivalent dose concept to plateau dose-response relationships. Radiat Environ Biophys 2005;44:235-9.

19. National Academy of Sciences. Health risks from exposure to low levels of ionizing radiation: BEIR VII phase 2. Washington, DC: The National Academies Press; 2006.

20. Statistics Korea. Complete life-table 2012 [Internet]. Daejeon: Statistics Korea; c2016 [cited 2016 Nov 20]. Available from: http://kostat.go.kr.

21. Luxton RW. Radiation nephritis: a long-term study of 54 patients. Lancet 1961;2:1221-4.

22. Tanaka H, Hayashi S, Ohtakara K, Hoshi H. Hepatic dysfunction after radiotherapy for primary gastric lymphoma. J Radiat Res 2013;54:92-7.

23. Lee IJ, Seong J, Koom WS, et al. Selection of the optimal radiotherapy technique for locally advanced hepatocellular carcinoma. Jpn J Clin Oncol 2011;41:882-9.

24. Chen $D$, Wang $R$, Meng $X_{1}$, et al. A comparison of liver protection among 3-D conformal radiotherapy, intensitymodulated radiotherapy and RapidArc for hepatocellular carcinoma. Radiat Oncol 2014;9:48.

25. Berrington de Gonzalez A, Curtis RE, Kry SF, et al. Proportion of second cancers attributable to radiotherapy treatment in adults: a cohort study in the US SEER cancer registries. Lancet Oncol 2011;12:353-60.

26. Hall EJ. Intensity-modulated radiation therapy, protons, and the risk of second cancers. Int J Radiat Oncol Biol Phys 2006;65:1-7. 